\title{
A four-parameters model for fatigue crack growth data analysis
}

\author{
M. Grasso, F. Penta, P. Pinto, G.P. Pucillo \\ Università di Napoli Federico II, Dipartimento di Ingegneria Industriale, P.le V. Tecchio 80 - 80125 Napoli, Italy \\ marzio.grasso@unina.it
}

\begin{abstract}
A four-parameters model for interpolation of fatigue crack growth data is presented. It has been validated by means of both data produced by the Authors and data collected from Literature. The proposed model is an enhanced version of a three-parameters model already discussed in a previous work that has been suitably modified in order to overcome some drawbacks raised when applied to a quite wider experimental data set. Results of validation study have also revealed that the new model, besides interpolating accurately crack growth data, allows to identify the presence of anomalies in the data sets. For this reason, by a suitable filter to be chosen depending on the size and number of anomalies, it can be used to remove them and obtain sigmoidal crack propagation curves smoother than those obtained when the current analysis techniques are used. In the end, possible model parameters correlations are analysed.
\end{abstract}

KEYWORDS. Fatigue crack propagation; Crack growth data analysis; Correlation model; Fatigue damage; Fatigue.

\section{INTRODUCTION}

I $\mathrm{t}$ is well known that the assessment of fatigue damage by means of phenomenological models is closely linked to the procedure and accuracy with which experimental raw crack growth data are analysed. Very often they are discontinuous and naturally irregular and their analysis has to be made on different levels to reach the model formulation or, at least, the canonical graphical correlation between the crack growth rates and the stress intensity factor range, which is the parameter controlling the phenomenon. Actually, when experimental results are analysed, in addition to the normal scatter due to both the phenomenon random character and measurement uncertainty, some further anomalies are usually observed in the homogeneous sequences of data points of the crack growth curves: significant deviations, apparently inconsistent and absolutely uncontrollable, of some data from the trend followed by all the remaining experimental points of the same curve. They are probably due to the random evolution, in the infinite points of the crack front, of the local combinations of stress states and material strengths. Thus, pursuing an improvement in the analysis of crack growth data would mean to formulate a correlation model able to reproduce more accurately the aforementioned trend and not only simply and solely improve the data points interpolation by new averaging techniques and alternative fitting methods.

The Standard procedures currently in use to carry out both fatigue crack propagation tests and data analysis do not guarantee that the anomalies in acquired data are absent and do not provide a mean nor a criterion to filter them [1]. The main analytical models found in Literature [2-8], based on polynomial or exponential interpolation formula, do not seem having solved completely the problem. Consequently, to improve the quality of raw crack propagation data analysis we propose an interpretative model whose validity has been checked with a very wide set of crack growth data. A similar attempt, reported in [9] and concerning a three parameters model, has been successfully tested using only experimental data produced by Ghonem and Dore [10]. However, this model revealed some limitations when used to interpolate both 
data produced by Virkler and co-workers [11] and those recently produced by Wu and Ni [12]. For this reason the model has been modified introducing two more parameters. The positive verification of the new model by means of both the cited data and those discussed in [13] closes the paper.

\section{EXPERIMENTAL DATA ANALYSED}

7 he methods developed to interpolate crack propagation data, besides those proposed by the ASTM Standard [1], are essentially based on the preliminary choice of a technique or a method to interpret the experimental raw data, i.e. crack length values as function of time or load cycles, to establish a correlation between the observed variables. Generally, they essentially are local interpolation methods of the data, which are not able to filter the irregularities or anomalies characterizing this type of measurements. Since the evolution of the propagation phenomenon is progressively faster when the crack approaches the critical condition, propagation models built on results obtained from a limited number of tests not only have a validity range closely linked to the particular experimentation carried out, but they are also not able to fit all crack growth data with the same accuracy for the whole field of cycles number of each test [9].

In order to work out a new analytical model of the link between crack length and the corresponding number of cycles overcoming the drawbacks observed with previous models, several fatigue crack growth data sets obtained with different materials, loading conditions and type of specimens have been analyzed and interpolated. In the following sections the main aspects of the testing activities carried out by authors of the different data sources are reported.

\section{Ghonem and Dore data [10]}

To analyse the validity of their Markov Chain crack growth model, Ghonem and Dore carried out tests at room temperature using $\mathrm{M}(\mathrm{T})$ specimens made of aluminium alloy 7075-T6 having the same thickness $(3.175 \mathrm{~mm})$ of the sheet from which they were extracted. The crack direction was perpendicular to the rolling direction of the sheet. Loading conditions applied after the precracking phase are reported in Tab. 1. N. 60 specimens were been tested under each loading level.

\begin{tabular}{lllll}
\hline Data set & $\mathrm{P}_{\max }(\mathrm{kN})$ & $\mathrm{P}_{\min }(\mathrm{kN})$ & $\Delta \mathrm{P}(\mathrm{kN})$ & $\mathrm{R}$ \\
Test I & 22.79 & 13.68 & 9.11 & 0.6 \\
Test II & 22.25 & 11.13 & 11.12 & 0.5 \\
Test III & 15.19 & 6.08 & 9.11 & 0.4 \\
\hline
\end{tabular}

Table 1: Loading Conditions related to Ghonem and Dore tests.

Virkler et Al. data [11]

Experimental activity of Virkler et Al. was aimed at:

$>$ determining which crack growth rate calculation method yields the least amount of error when the crack growth rate curve is integrated back to obtain the original a versus $\mathrm{N}$ curve data;

$>$ determining the statistical distribution of $\mathrm{N}$ as a function of the crack length;

$>$ determining the statistical distribution of $\mathrm{da} / \mathrm{dN}$ as a function of $\Delta \mathrm{K}$;

$>$ determining the variance of a set of $a$ versus $N$ curves predicted from the $\mathrm{da} / \mathrm{dN}$ distribution parameters.

Crack growth testing was carried out on a sample of n.68 M(T) specimens, $558.8 \mathrm{~mm}$ long and $152.4 \mathrm{~mm}$ wide, made of aluminium alloy 2024-T3 and extracted from a sheet having thickness equal to $2.54 \mathrm{~mm}$. The crack starter was machined with an electro-discharge machine. All tests were conducted under the same experimental conditions and with the loading level reported in Tab. 2.

\begin{tabular}{ccccc}
\hline & $\mathrm{P}_{\max }$ (kip) & $\mathrm{P}_{\min }$ (kip) & $\Delta \mathrm{P}$ (kip) & $\mathrm{R}$ \\
Test I & 5.25 & 1.05 & 4.2 & 0.2 \\
\hline
\end{tabular}

Table 2: Loading Conditions related to Virkler tests. 
Moreover, to study the statistical distribution of the number of cycles $N(a)$ to reach a specific value of the crack length $a$, data acquisition was carried out fixing preliminary a set of $a$ values and recording then the cycles number spent by the cracks to reach them.

Wu and Ni data [12]

The experimental activity of $\mathrm{Wu}$ and $\mathrm{Ni}$ was carried out on $\mathrm{n} .3$ samples of compact tension $\mathrm{C}(\mathrm{T})$ specimens made of aluminium alloy 2024-T351, having thickness $B=12 \mathrm{~mm}$ and width $\mathrm{W}=50 \mathrm{~mm}$. Tests were carried out with variable amplitude loading for one sample, whereas for the other two samples, marked as CA1 and CA2 and composed of 30 and 10 specimens respectively, constant amplitude loadings of Tab. 3 were used.

\begin{tabular}{lllll}
\hline & $\mathrm{P}_{\max }(\mathrm{kN})$ & $\mathrm{P}_{\min }(\mathrm{kN})$ & $\Delta \mathrm{P}(\mathrm{kN})$ & $\mathrm{R}$ \\
CA1 & 4.5 & 0.9 & 3.6 & 0.2 \\
CA2 & 6.118 & 3.882 & 2.236 & 0.63 \\
\hline
\end{tabular}

Table 3: Loading Conditions related to Wu \& Ni tests.

The main purpose of this work was to acquire enough experimental data to study the effects of different loading levels on the variability of fatigue crack growth data.

GPP data [13]

A number of crack growth tests were carried out at the Materials and Structures Mechanics Laboratory of the Department of Industrial Engineering on types of rail steel, obtaining a sample of $\mathrm{n}^{\circ} 21$ crack growth curves. SEN(B) specimens, having thickness $\mathrm{B}=20 \mathrm{~mm}$ and height $\mathrm{W}=45 \mathrm{~mm}$, were used in the three point bending configuration. Loading conditions are listed in Tab. 4.

\begin{tabular}{lllll}
\hline Material & $\mathrm{P}_{\max }(\mathrm{kN})$ & $\mathrm{P}_{\min }(\mathrm{kN})$ & $\mathrm{DP}(\mathrm{kN})$ & $\mathrm{R}$ \\
Rail steel & 12.03 & 6.015 & 6.015 & 0.5 \\
\hline
\end{tabular}

Table 4: Loading Conditions related to GPP tests

\section{MODEL FORMULATION}

$\mathrm{I}$

n order to formulate a new model able to overcome the aforementioned problems, all the available data set have been analysed. The large number and the diversity of the collected data should give to the model the value of a reliable tool to interpolate fatigue crack growth data obtained from a wide class of materials, with different specimens and loading conditions.

The starting point of our study has been the three-parameters model discussed elsewhere [9]. By means of a trial and error method, its mathematical expression has been modified and further parameters have been introduced in it with the aim of identifying an equation of the $a(N)$ curve able to interpolate all analysed data. The final result is the following fourparameters model

$$
a(N)=b \cdot \tau^{p}+k \cdot e^{\left(\frac{\tau^{\alpha}}{\beta-\tau^{\alpha}}\right)}
$$

where

$$
\tau=\frac{N+N_{0}}{N_{f}+N_{0}}
$$

the functions $b$ and $k$ have the expressions

$$
b=a_{f}-k \cdot e^{\frac{1}{\beta-1}}
$$




$$
k=\frac{a_{0}-a_{f}\left(\frac{N_{0}}{N_{0}+N_{f}}\right)^{p}}{e^{\left(\frac{\left(\frac{N_{0}}{N_{f}+N_{0}}\right)^{\alpha}}{\beta-\left(\frac{N_{0}}{N_{f}+N_{0}}\right)^{\alpha}}\right)}-\left(\frac{N_{0}}{N_{f}+N_{0}}\right)^{p} \cdot e^{\left(\frac{1}{\beta-1}\right)}}
$$

and where $a_{0}$ and $a_{f}$ are respectively the initial and final crack lengths, $\mathrm{N}_{\mathrm{f}}$ is the number of cycles to failure and $N_{0}, \alpha, \beta$ and $p$ are four parameters to be determined by least-square method.

By means of the Eq. (1), the accuracy of which will be discussed later, the crack growth rate can be continuously evaluated in the whole range $\mathrm{N}_{\text {initial }}-\mathrm{N}_{\text {final, }}$ without magnifying both the irregularities and anomalies of the raw data, nor loosing information in the initial and final parts of the experimental range of the number of cycles, as it happens when a procedure similar to those proposed by the ASTM is used [1]. Moreover, once the parameters model have been identified, it is possible to extrapolate the curve outside the range of the number of cycles in which crack lengths were recorded.

\section{MODEL VERIFICATION}

he different raw data sets used to validate the proposed model are shown in Fig. 1 and 2.

Best fitting of eq. (1) has been carried out for all the crack growth curves of all the data sets. To quantify the goodness-of-fit of the eq. (1), the coefficient of determination $\mathrm{R}^{2}$ has been computed for each curve. For most of them the $\mathrm{R}^{2}$ values were higher than 0.999 . Only with the crack path $\mathrm{n}^{\circ} 29$ of the set III of the Ghonem and Dore data a value less than 0,975 was obtained, due to the presence of remarkable irregularities in the path.

Some raw data together with the corresponding best fitting curve obtained with the proposed model are shown in Fig. 35. It is evident the accuracy with which the proposed model is able to interpolate the experimental data points.
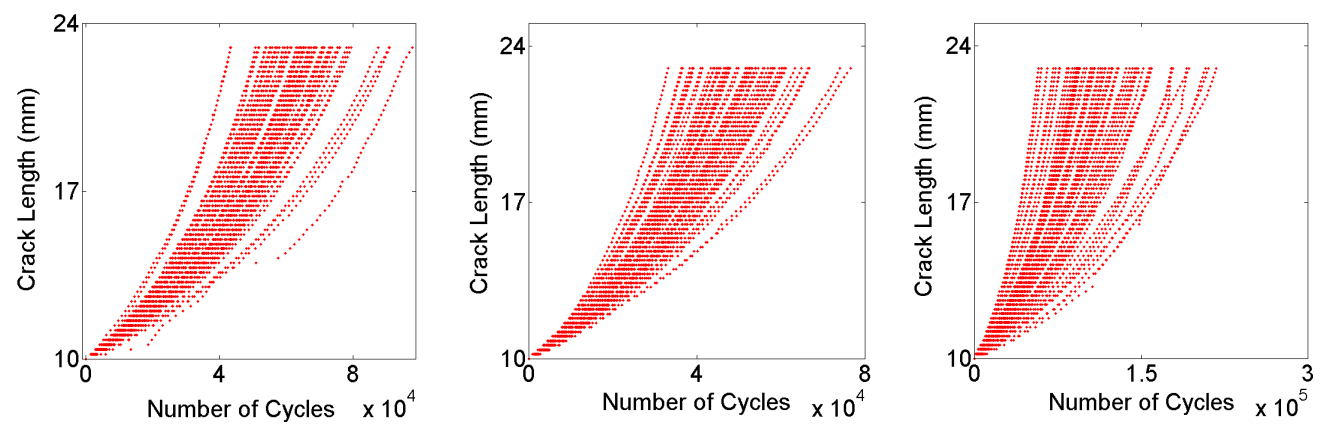

Figure 1: Experimental data produced by Ghonem and Dore: set I (left), set II, set III (right).
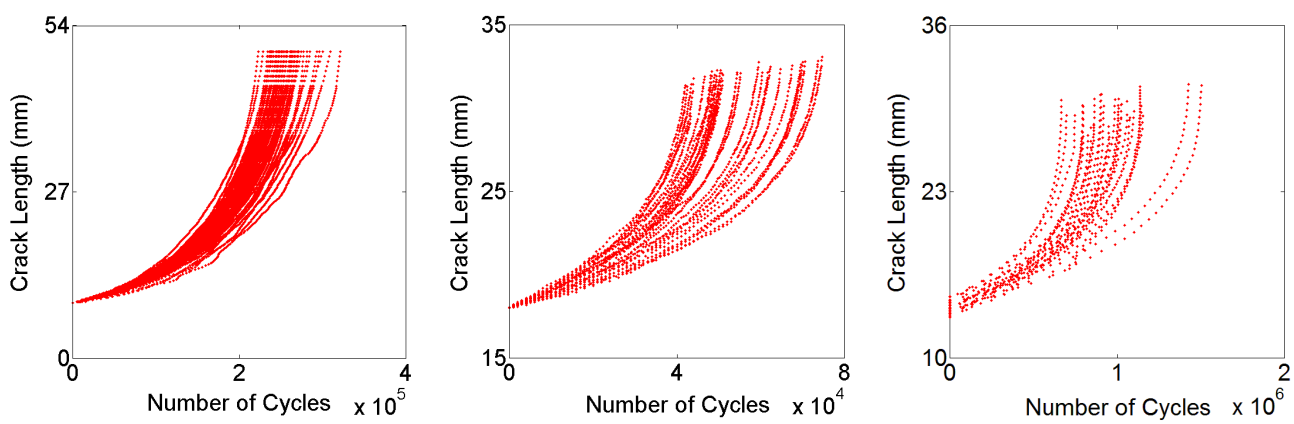

Figure 2: Experimental data produced by: Virkler (left), Wu \& Ni, GPP (right). 

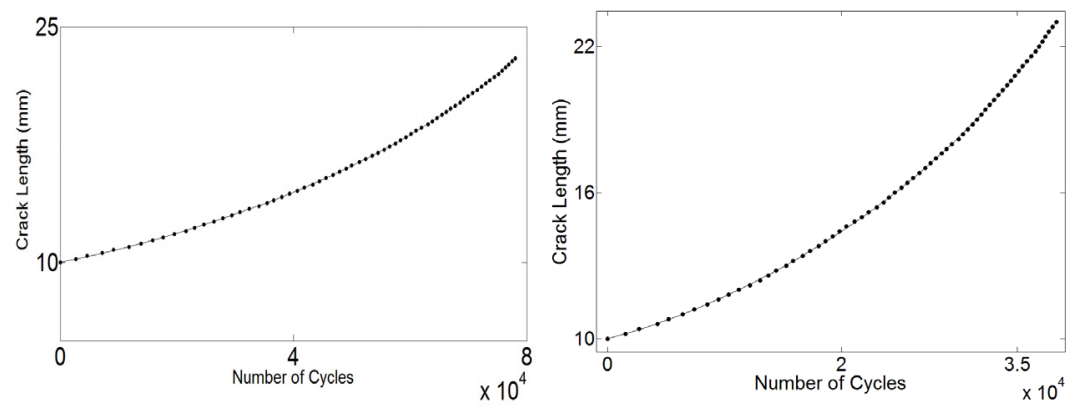

Figure 3: Experimental data produced by Ghonem and Dore: set I (left) and set II (right) together with the fitting curves.
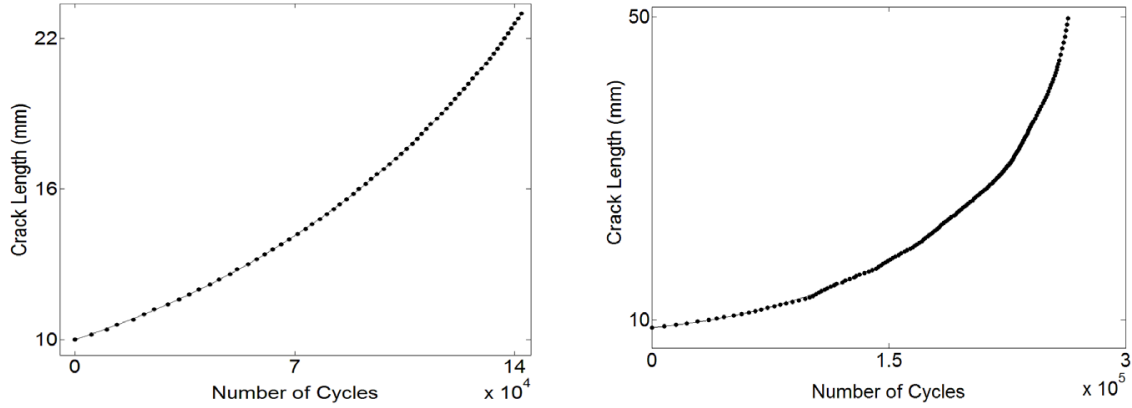

Figure 4: Experimental data produced by Ghonem and Dore: set III (left) and Virkler (right) together with the fitting curves.
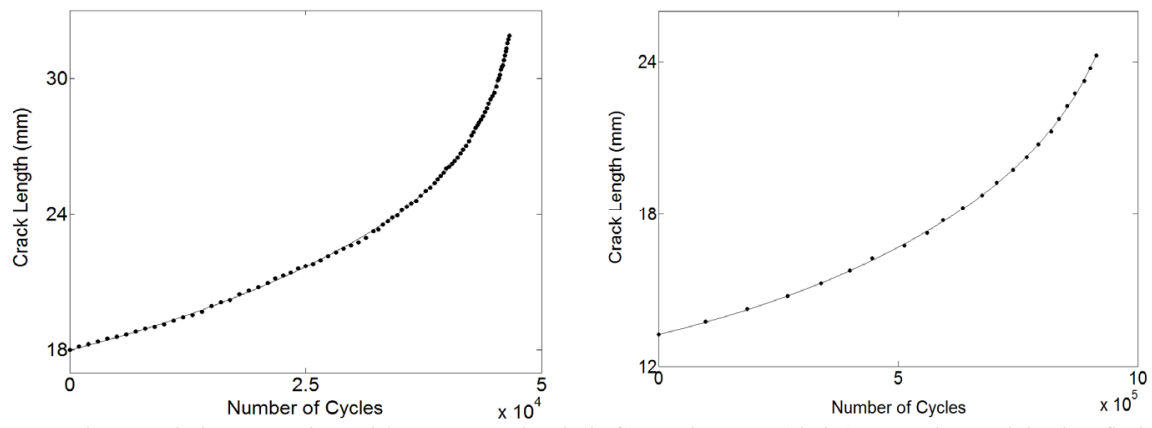

Figure 5: Experimental data produced by Wu and Ni (left) and GPP (right) together with the fitting curves.
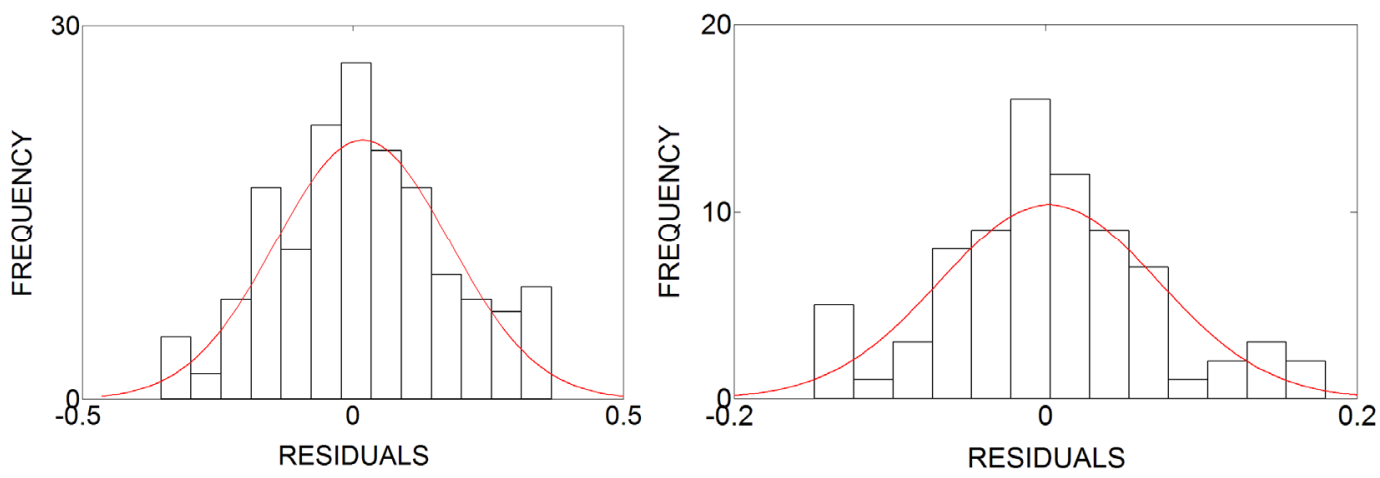

Figure 6: PDF of residuals.

Normality of the residuals obtained from each curve has been verified by the $\chi^{2}$ normality tests and the corresponding residuals frequency histograms have been also produced. In Fig. 6, the residuals probability density functions estimated for two curves, one from Virkler set and one from Wu and Ni set, are reported, whereas means and standard deviations of residuals of all curves are shown in Fig. 7 - 12. 

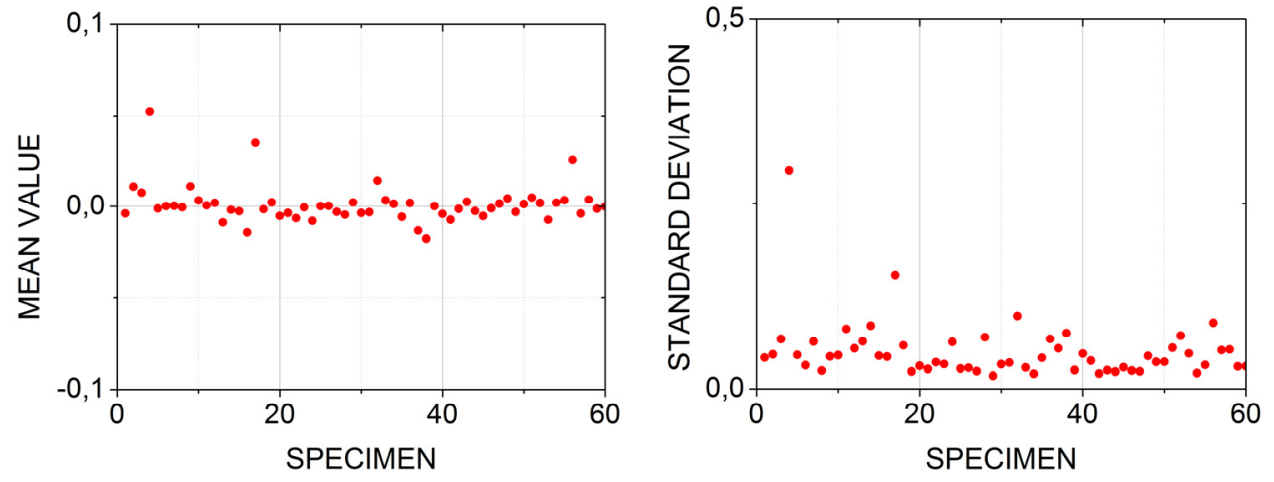

Figure 7: Mean (left) and standard deviation (right) of residuals obtained by fitting Ghonem and Dore set I.
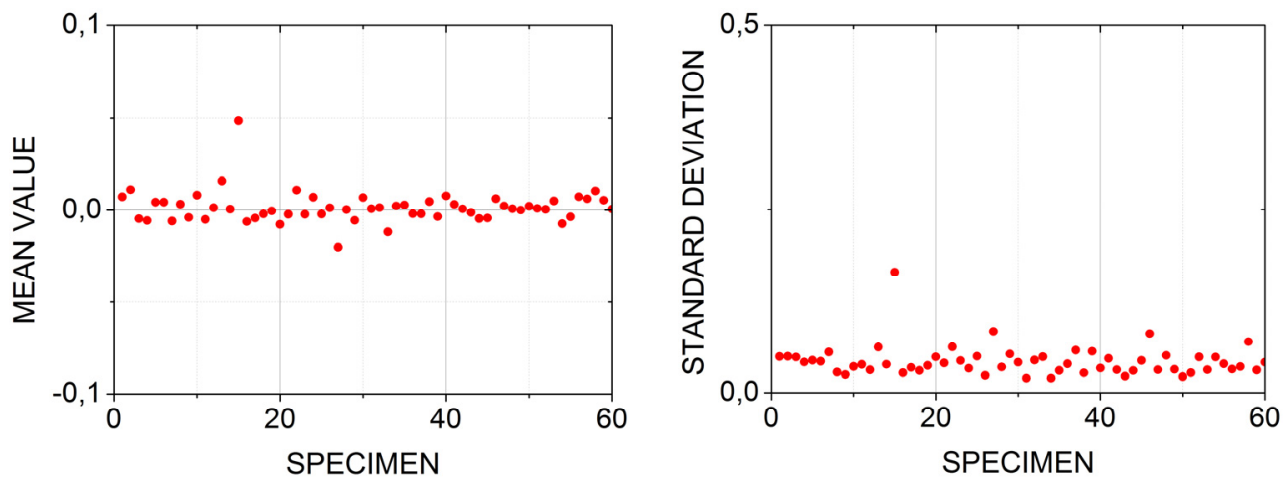

Figure 8: Mean (left) and standard deviation (right) of residuals obtained by fitting Ghonem and Dore set II.
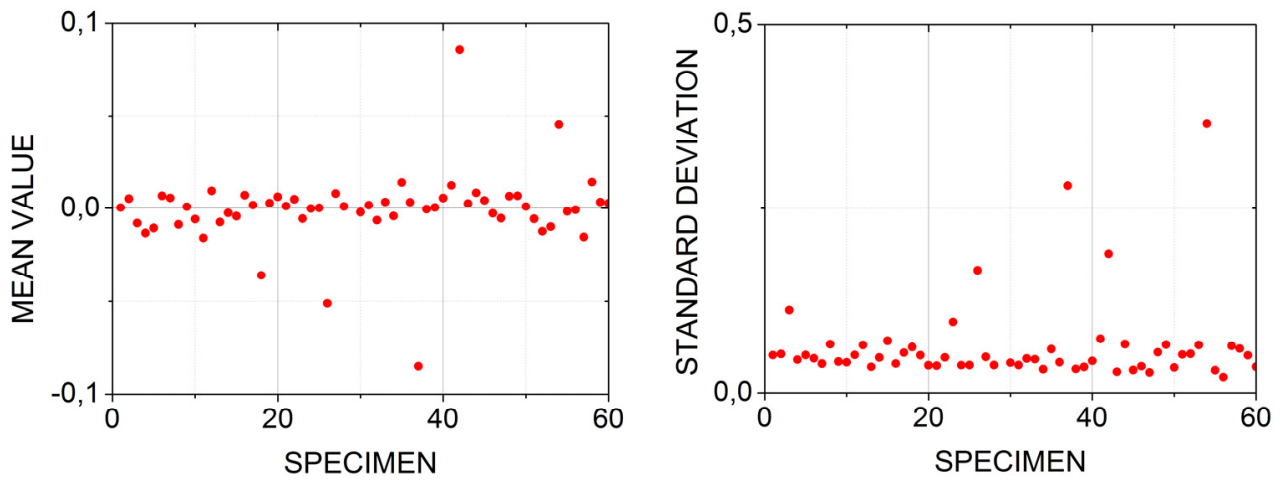

Figure 9: Mean (left) and standard deviation (right) of residuals obtained by fitting Ghonem and Dore set III.
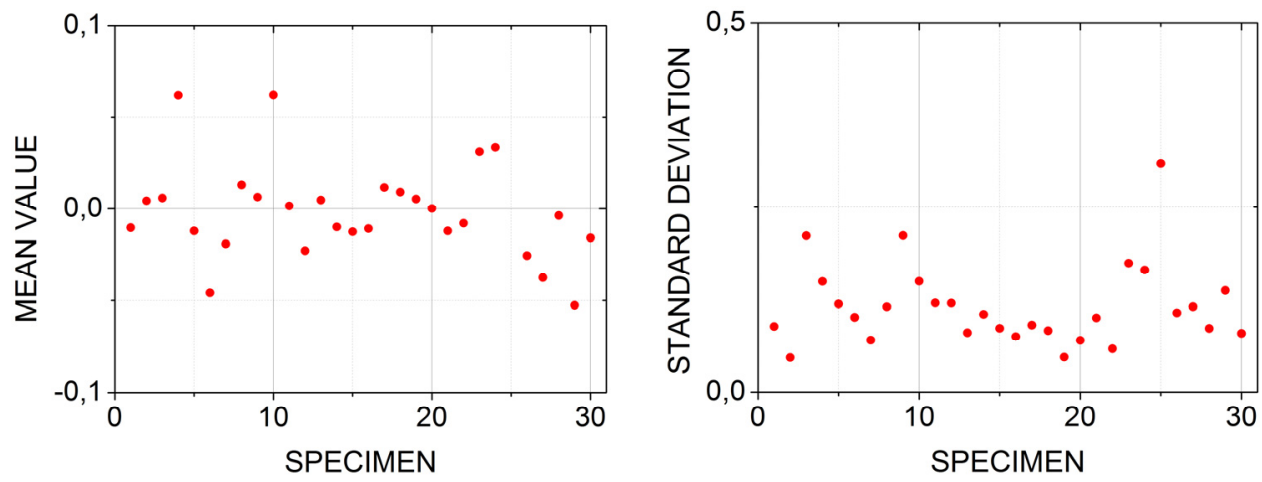

Figure 10: Mean (left) and standard deviation (right) of residuals obtained by fitting GPP data set. 

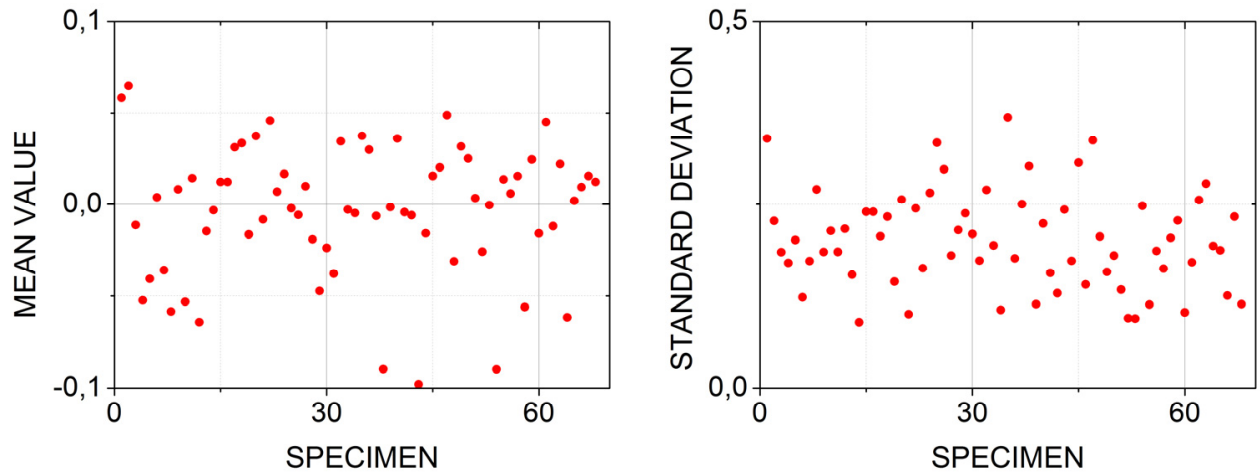

Figure 11: Mean (left) and standard deviation (right) of residuals obtained by means of fitting the Virkler data set.
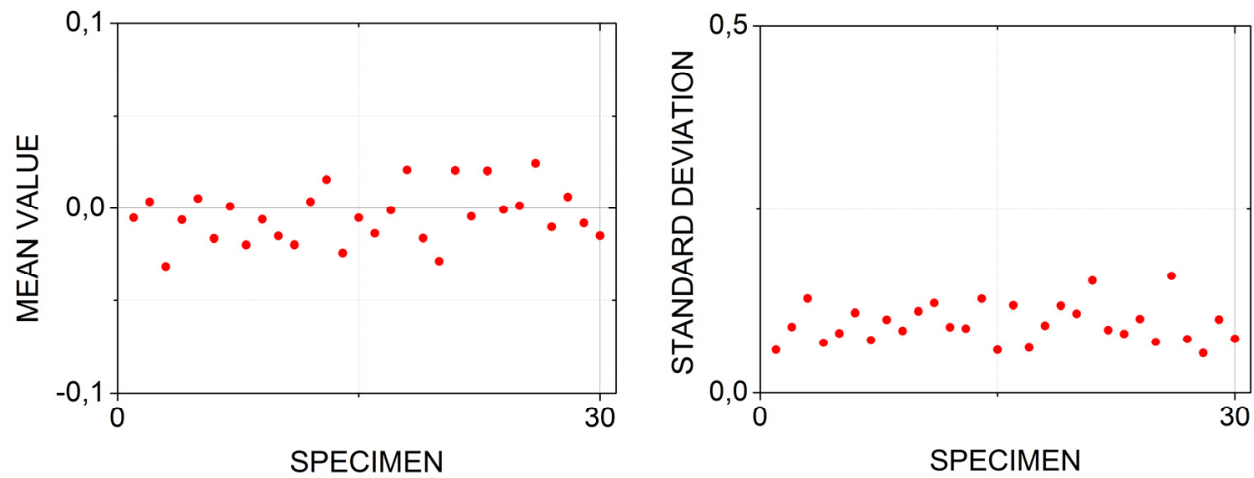

Figure 12: Mean (left) and standard deviation (right) of residuals obtained by fitting Wu and Ni data set.

The results of normality tests listed in Tab. 5 highlight that for a large part of the experimental curves analysed in the present study, the residuals have a normal distribution with a confidence level of $95 \%$. However, in some cases the normality hypothesis has to be refused, due to some anomalies in the raw data that are evident also in the residuals frequency histogram. As already discussed elsewhere [9], when the experimental crack growth curve is compared with the best fitting curve on an enlarged scale, it is possible recognize that the anomalies observed in frequency histograms of the residuals are due to experimental data points moving away from the characteristic trend of the remaining points of the same curve.

\begin{tabular}{cccc}
\hline & & Accepted & Refused \\
GHONEM & set_I & 34 & 26 \\
\& DORE & set_II & 45 & 15 \\
GPP & set_III & 43 & 17 \\
Wu \& Ni & & 21 & $/ /$ \\
VIRKLER & & 35 & 33 \\
\hline
\end{tabular}

Table 5: Results of Normality Tests.

Similar conclusions can be drawn for the outliers present in the graphs of the means and standard deviations of the residuals reported in Fig. 7 - 12. Indeed, they are due to either singular raw data that move away from the common trend or groups of data lying on a trend curve that is different, for a short part, from the trend curve of the remaining data points. These anomalies change the response of the least-square method used to identify the model parameters. Due to their presence, the algorithm gives parameters values defining a fitting curve with an asymmetric distribution of residuals and a mean value significantly far from zero. In order to point out what previously stated, two cases of curves having irregular groups of data points, an asymmetric distribution of the residuals and an unbalanced mean are reported in Fig. 13 $-15$. 


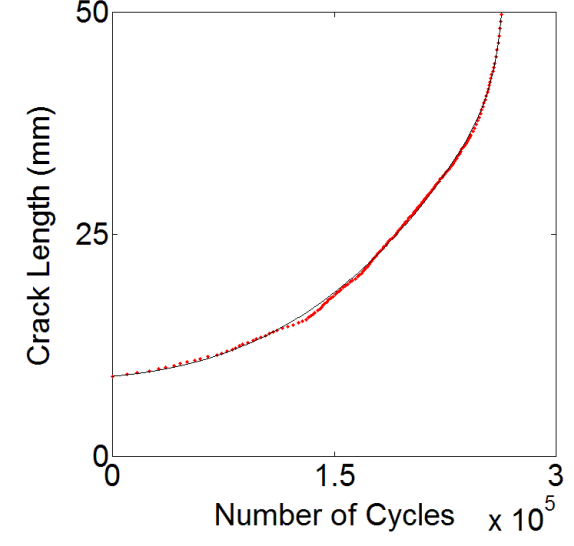

Figure 13: Curve fitting of Virkler data set.

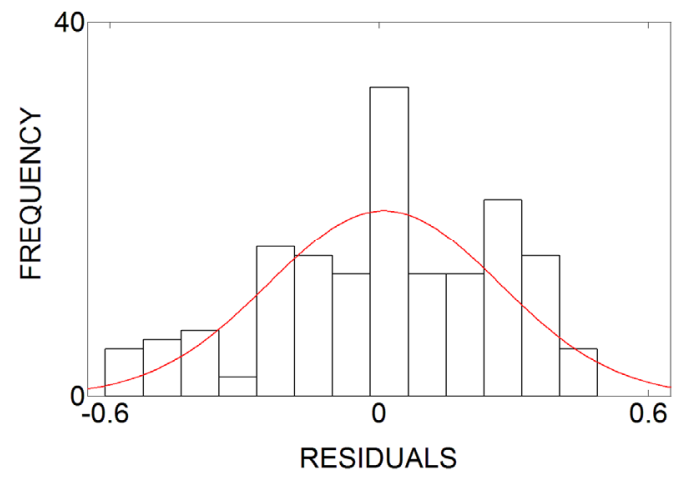

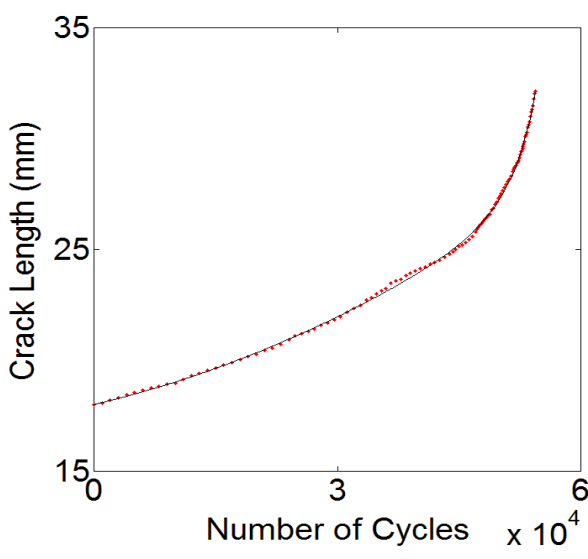

Figure 14: Curve fitting of Wu \& Ni data set.

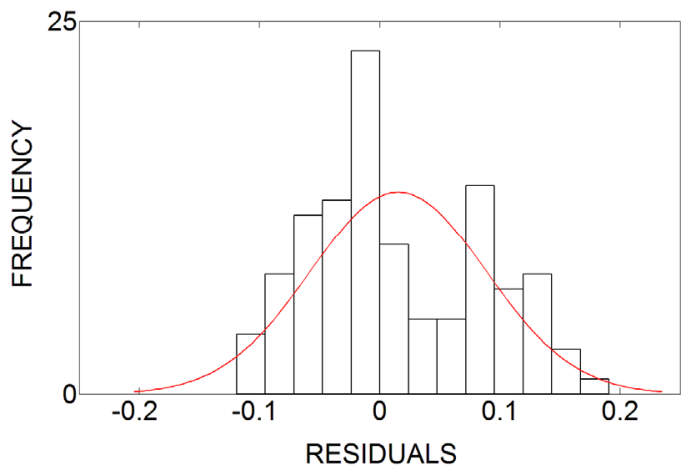

Figure 15: PDF of residuals

Despite the anomalies, the interpolations carried out by means of the proposed model are visually acceptable in both cases and the corresponding coefficient of determination $\mathrm{R}^{2}$ is close to one.

It can be inferred that, if the residuals distribution is not normal, it is due to the presence of anomalous experimental data points. Therefore, what may seem a limit of the proposed model in fitting the data points, actually can be used as a tool to verify if anomalous points sequences are present in a set of experimental data.

When this happens, a suitable filter, to be chosen depending on the size and the number of anomalies in the data set to be analysed, can be identified to remove all data points moving away in an anomalous and excessive way from the trend followed by the other data points of the same set that has to be unique as it is unique the physical law that characterize the fatigue crack growth phenomenon in each material free of geometrical singularities and microstructural inhomogeneity.
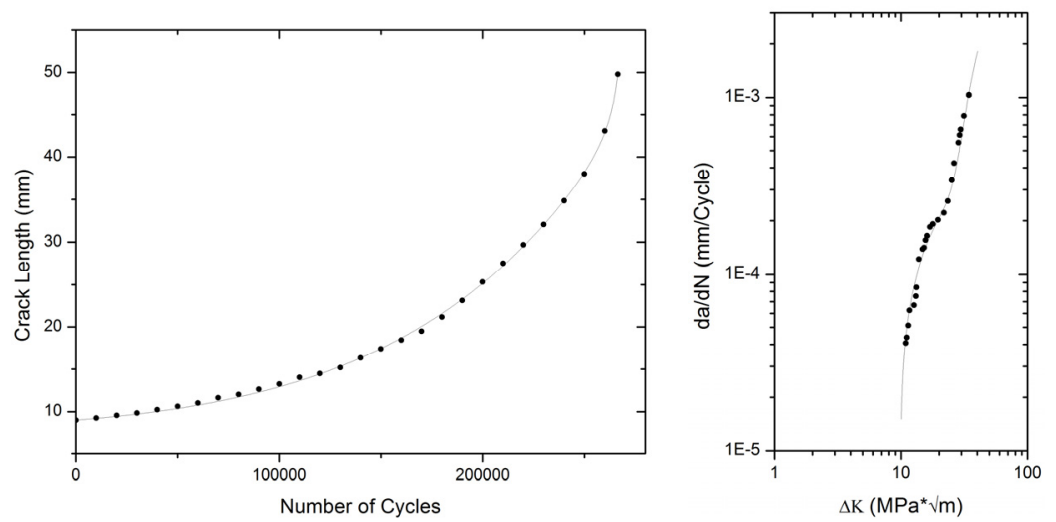

Figure 16: Fitting of one of the Virkler data set curve (left) FCG curve evaluated in accordance with the ASTM practice and that with the proposed model (right). 
As reported in Fig. 16, a model built up in this way allows, by means of its analytical derivative, to determine a fatigue crack growth curve that is continuous, regular and defined in the whole $\Delta \mathrm{K}$ field, from the threshold value to the critical one. A sigmoidal crack rate curve obtained using the proposed model is compared to the one obtained using the practice suggested by the ASTM Standard in Fig. 16. Experimental data used for this comparison are from one of the crack path obtained by Virkler and are plotted in fig 16 together with the best fitting curve of the proposed model.

It is evident that when crack growth data are analysed using the four-parameters model the information gathered from the material testing activity is maximized, improved and fully exploited.

\section{COMPARISON BETWEEN TWO MODELS PROPOSED BY THE AUTHORS}

$\mathrm{I}$

$\mathrm{t}$ seems proper to report a short comparison between the capability of the four-parameters model here discussed and the three-parameters model [9] from which the new model has been derived, in order to highlight analogies and differences.

A first comparison can be done analyzing the graphs reported in Fig. 16 where raw data selected from the sets produced by Ghonem and Dore are plotted together with the fitting curves obtained using the two models. It can be seen the goodness-of-fit of both models. However, the four-parameters model is able to get closer to the trend of the experimental data.
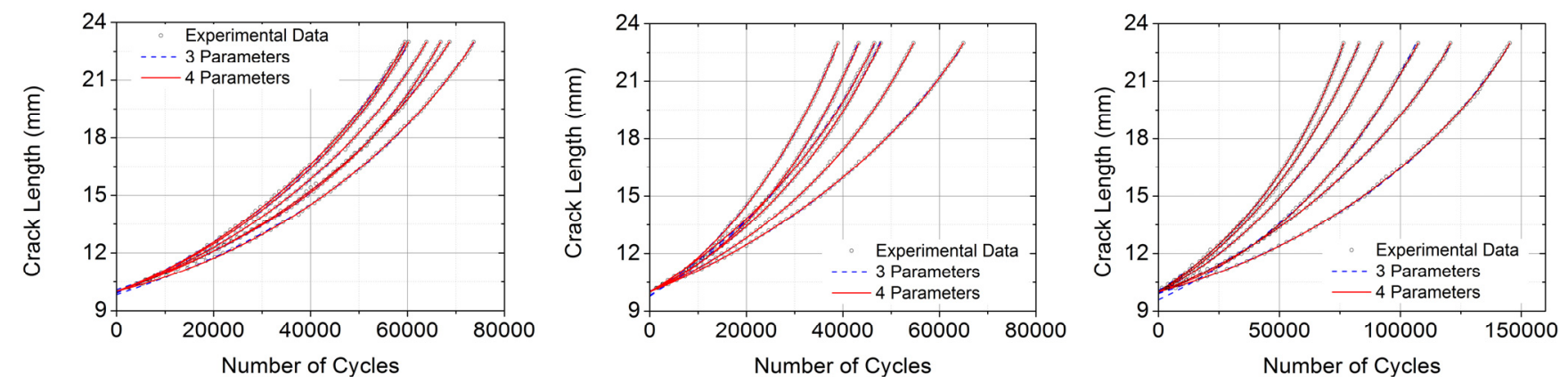

Figure 17: Fitting of Ghonem and Dore data set using the three-parameters model (dash line) and the four-parameters model (solid line)

By inspection of Fig. 17, where experimental data produced by Virkler, Wu and Ni and GPP together with the corresponding fitting curves are reported, the limits of the three-parameters model and the accuracy of the four-parameter model in fitting these data are noted.
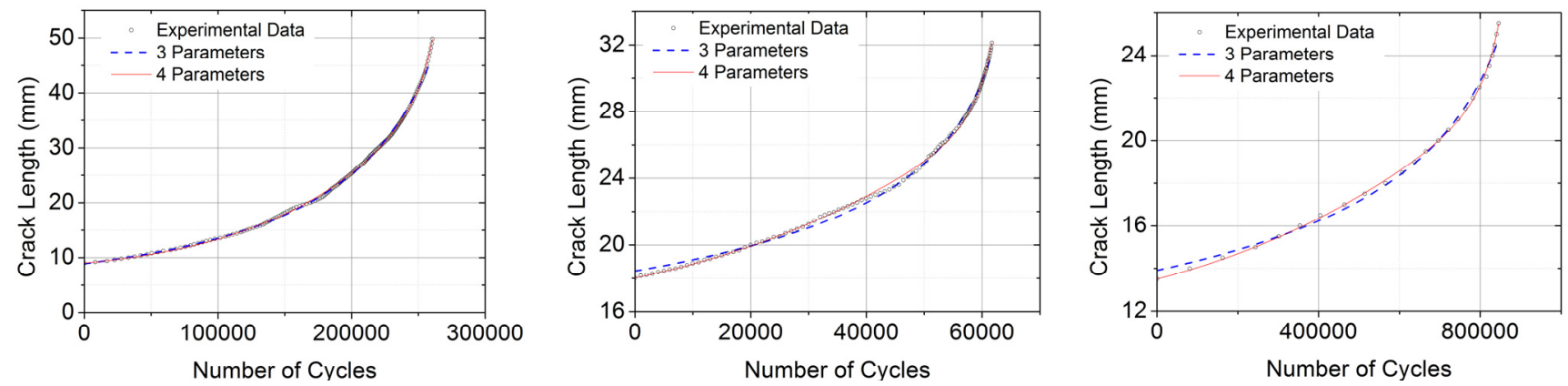

Figure 18: Fitting of Virkler, Wu and Ni and GPP data sets using the three-parameters model (dash line) and the four-parameters model (solid line).

\section{CORRELATION BETWEEN MODEL PARAMETERS}

$\mathrm{I}$ $\mathrm{n}$ order to evaluate the possibility of reducing the number of independent parameters of the proposed model, correlation tests between the values of the parameters have been carried out. For this purpose Kendall correlation test has been used [14] with the limitation of accepting the only correlations that verify the hypothesis of "strong 
correlation", which is having characteristic coefficient $\varrho$ in the range $0.7-1$.

The model parameters pairs that stand the test for all considered data sets are reported in Fig. 18, 19 and 20.

Obviously, it is necessary to further investigate these statistical relationships by means of a wide experimental activity and considering more materials and loading conditions.
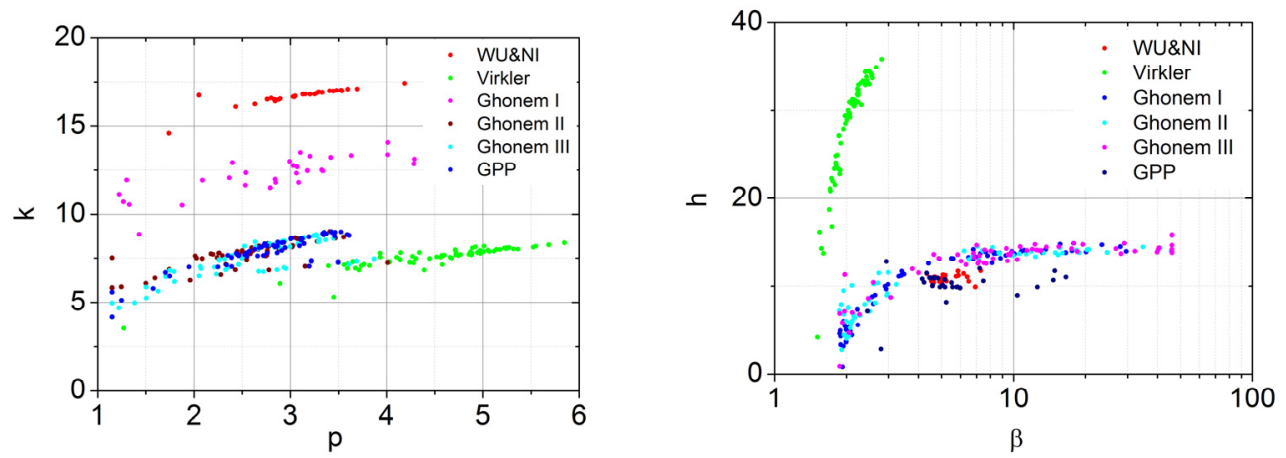

Figure 19: Parameters correlations between $\mathrm{p}$ and $\mathrm{k}$ (left) and $\beta$ and $\mathrm{h}$ (right).
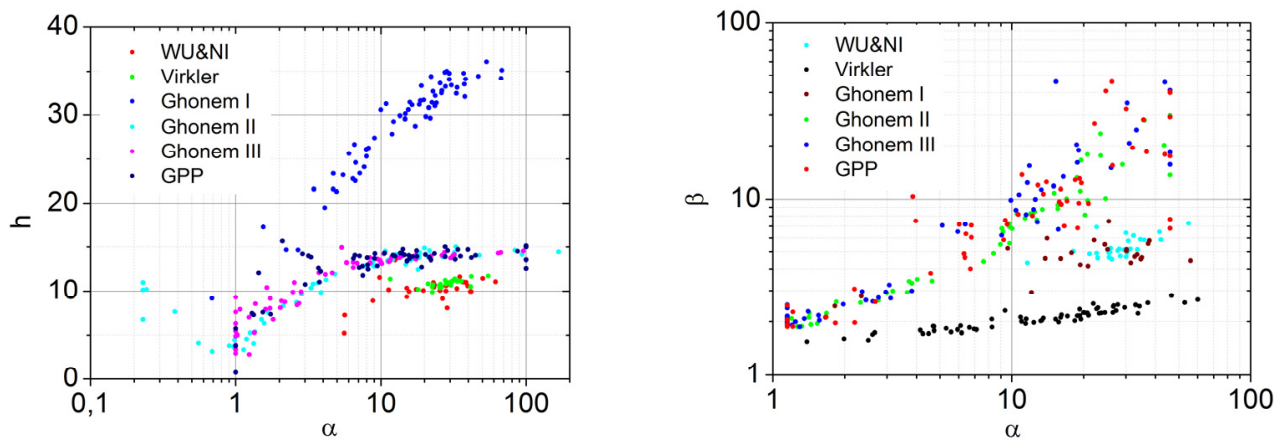

Figure 20: Parameters correlations between $\alpha$ and h (left), $\alpha$ and $\beta$ (right).

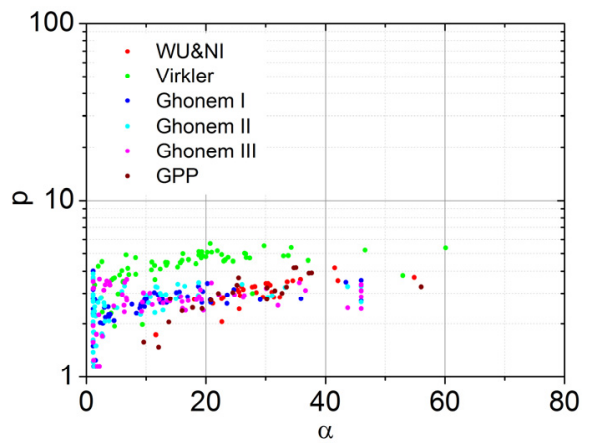

Figure 21: Parameters correlation between $\alpha$ and $\mathrm{p}$.

\section{CONCLUSIONS}

$\mathrm{B}$ $y$ the analysis of several sets of fatigue crack growth curves, a four-parameter model for the interpolation of crack propagation data has been derived. The proposed model is alternative to the analysis techniques proposed by the ASTM E647 Standard and it represents an enhancement of another model presented in a previous paper.

It has been discussed the ability of the new model in fitting, with the needed accuracy, a wide range of experimental data produced with different specimen geometries, different materials and different loading condition.

Moreover, it has been highlighted that it is possible to identify, by means of cross checks carried out using the normality tests of the residuals and the correlation coefficient $\mathrm{R}^{2}$, those groups of data points moving away in an anomalous and irregular way from the trend of all other data points, to remove them from the sample of results. 
In the end, the model parameters have been identified and some interesting correlations between them have been found. These latter, by further analysis and validation on the basis of a wider experimentation results could be usefully adopted to build up a new phenomenological model for fatigue crack growth that is robust, adaptable and valid in the full crack propagation field.

\section{REFERENCES}

[1] ASTM E 647: Standard test method for measurement of fatigue crack growth rates, USA, (2011).

[2] Mukherjee, B., A note on the analysis of fatigue crack growth data, Int. J. of Fracture, 8(4) (1972) 449-452.

[3] Smith, R. A., The determination of fatigue crack growth rate from experimental data, Int. J. of Fracture, 9 (1973) $352-$ 355.

[4] Davies, K.B., Feddersen, Evaluation of fatigue-crack growth rates by polynomial curve fitting, Int. J. of Fracture, 9 (1973) 116-18.

[5] Polak, J., Knesl, Z, On the fatigue crack growth rate evaluation from experimental data, Int. J. of Fracture, 11, (1975), 693-96.

[6] De Iorio, A., Ianniello, D., Penta, F., Santoro, E., In: $8^{\text {th }}$ ECCM, Naples, Italy, (1998).

[7] Zheng, J., Powell, B. E., A method to reduce the scatter in fatigue crack growth rate data, Fatigue Fract. Engng Mater. Struct., 20(9) (1997) 1341-1350.

[8] Mohanty, J.R., Verma, B.B., Ray, P.K., Determination of fatigue crack growth rate from experimental data: a new approach", Int. J. Microstructure and Materials Properties, 5(1) (2010) 79-87.

[9] De Iorio, A., Grasso, M., Penta, F., Pucillo, G.P., A three-parameter model for fatigue crack growth data analysis, Frattura ed Integrità Strutturale, 21 (2012) 21-29.

[10] Ghonem, H. Dore, S., Experimental study of the constant-probability crack growth curves under constant amplitude loading, Engineering Fracture Mechanics, 17(I) (1987) 1-15.

[11] Virkler, D. A., Hillberry, B. M., Goel, P. K., The Statistical Nature of Fatigue Crack Propagation, Transactions of the ASME, 101 (1979) 148-153.

[12] Wu, W.F., Ni, C.C., Statistical aspects of some fatigue crack growth data, Engineering Fracture Mechanics, 74 (2007) 2952-2963.

[13] De Iorio, A., Grasso, M. Penta, F., Pucillo, G.P., About the certification of railway rails, Frattura ed Integrità Strutturale, 26 (2014) 57 - 68.

[14] Kendall, M. G., Rank correlation methods, (2nd ed.). New York, Hafner, (1955). 